PROCEEDINGS OF THE

AMERICAN MATHEMATICAL SOCIETY

Volume 135, Number 6, June 2007, Pages 1929-1934

S 0002-9939(07)08653-4

Article electronically published on January 12, 2007

\title{
MODEL THEORY OF PARTIAL DIFFERENTIAL FIELDS: FROM COMMUTING TO NONCOMMUTING DERIVATIONS
}

\author{
MICHAEL F. SINGER
}

(Communicated by Julia Knight)

\begin{abstract}
McGrail (2000) has shown the existence of a model completion for the universal theory of fields on which a finite number of commuting derivations act and, independently, Yaffe (2001) has shown the existence of a model completion for the univeral theory of fields on which a fixed Lie algebra acts as derivations. We show how to derive the second result from the first.
\end{abstract}

\section{INTRODUCTION}

In [3, McGrail gives axioms for the model completion of the universal theory of fields of characteristic zero with several commuting derivations. Independently, Yaffe [7] gave axioms for the model completion of the universal theory of a more general class of fields: $L D F_{0}$, the universal theory of fields of characteristic zero together with a finite-dimensional Lie algebra acting as derivations. The goal of this short note is to show that starting from McGrail's results one can quickly write down axioms for Yaffe's model completion, that is, one can reduce the noncommutative case to the commutative case.

Another axiomatization of the model completion of a theory of differential fields with noncommuting derivations has been given by Pierce [4] using differential forms and a version of the Frobenius Theorem. In fact, one can state the Frobenius Theorem as a result that allows one to replace noncommuting vector fields with commuting ones: given an involutive analytic system $\mathcal{L}_{y}, y \in M$, of tangent spaces of rank $p$ on an analytic manifold $M$ and a point $x \in M$, one can choose analytic coordinates $x_{1}, \ldots, x_{p}$ around $x$ such that $\mathcal{L}_{y}$ is spanned by $\left(\partial / \partial x_{1}\right)_{y}, \ldots,\left(\partial / \partial x_{p}\right)_{y}$ for all $y$ in an open set containing $x$ ( $c f$. [6], Theorem 1.3.3). In his introductory remarks, Pierce states that one might be able to reduce the noncommutative case to the commutative case ([4, pp. 924-925) but does not give details 1 proceeding rather to develop his results ab initio. The present note carries through this reduction.

Received by the editors November 25, 2005 and, in revised form, January 21, 2006.

2000 Mathematics Subject Classification. Primary 03C10; Secondary 35A05, $12 \mathrm{H} 05$.

The preparation of this article was partially supported by NSF Grant CCR-0096842.

${ }^{1}$ Note added in proof. The proof of Theorem 5.3 of 4 does give more of an indication as to how this can be done. It should also be noted that there are similarities between Lemmas 5.2 and 4.7 of [4] and Lemmas 2.1 and 2.2 of this paper, although the proofs are different.

(C)2007 American Mathematical Society Reverts to public domain 28 years from publication 


\section{Commuting BASES OF Derivations}

In [7], Yaffe defines the universal theory $L D F_{0}$ of Lie differential fields. To do this he fixes a field $\mathcal{F}$ of characteristic zero, a finite-dimensional $\mathcal{F}$-vector space $\mathcal{L}$ with a Lie multiplication making it a Lie algebra over a subfield of $\mathcal{F}$, and a vector space homomorphism $\phi_{\mathcal{F}}: \mathcal{L} \rightarrow \operatorname{Der}(\mathcal{F})$, the Lie algebra of derivations on $\mathcal{F}$, preserving the Lie multiplication. We fix a basis $\left\{D_{1}, \ldots, D_{n}\right\}$ of $\mathcal{L}$ and let $\left[D_{k}, D_{l}\right]=\sum_{m} \alpha_{k l}^{m} D_{m}$ for some $\alpha_{k l}^{m} \in \mathcal{F}$. The elements $\alpha_{k l}^{m}$ are called the structure constants of $\mathcal{L}$. The language for $L D F_{0}$ is the language of rings together with unary function symbols $D_{i}, i=1, \ldots, n$, and constant symbols for each element of $\mathcal{F}$. The theory $L D F_{0}$ consists of

- the diagram of $\mathcal{F}$ including the action of the $D_{i}$,

- the theory of integral domains of characteristic zero,

- axioms stating that the $D_{i}$ are derivations,

- for each $k, l$ an axiom of the form $\forall x\left(D_{k} D_{l} x-D_{l} D_{k} x=\sum_{m} \alpha_{k l}^{m} D_{m} x\right)$.

We shall refer to a model of $L D F_{0}$ as a Lie ring over $\mathcal{L}$ or, more simply, a Lie ring, if it is clear which $\mathcal{L}$ is used.

We will need another concept from [7]: the ring of normal polynomials. This is defined as follows. Let $\mathcal{A}$ be a model of $L D F_{0}$. For each $I=\left\langle i_{1}, \ldots, i_{n}\right\rangle \in \mathbb{N}^{n}$ define a variable $X_{I}$ and consider the ring $\mathcal{R}_{\mathcal{A}}=\mathcal{A}\left[\left\{X_{I}\right\}_{I \in \mathbb{N}^{n}}\right]$ of polynomials in this infinite set of variables. Yaffe shows ([7], pp. 57-61) how one can define an action of $\mathcal{L}$ on $\mathcal{R}_{\mathcal{A}}$ so that this ring is a model of $L D F_{0}$, and one sees from his proof that the $D_{i}$ act as linearly independent derivations. Although this is not needed in the following lemma, we note that Yaffe also shows that if $\mathcal{B}$ is a model of $L D F_{0}$ that extends $\mathcal{A}$ and $b \in \mathcal{B}$, then the map from $\mathcal{R}_{\mathcal{A}}$ to $\mathcal{B}$ given by $X_{I} \mapsto D^{I}(b)=D_{1}^{i_{1}} D_{2}^{i_{2}} \ldots D_{n}^{i_{n}}(b)$ for all $I$ is a homomorphism of structures. Yaffe's construction of $\mathcal{R}_{\mathcal{A}}$ allows us to conclude

Lemma 2.1. If $\mathcal{A}$ is a model of $L D F_{0}$ with derivations $D_{1}^{\mathcal{A}}, \ldots, D_{n}^{\mathcal{A}}$, then $\mathcal{A}$ embeds in a model $\mathcal{B}$ of $L D F_{0}$ with derivations $D_{1}^{\mathcal{B}}, \ldots, D_{n}^{\mathcal{B}}$ extending the derivations of $\mathcal{A}$ such that the $D_{1}^{\mathcal{B}}, \ldots, D_{n}^{\mathcal{B}}$ are linearly independent over $\mathcal{B}$.

To show that the axioms in the next section yield a model completion of this theory we need two additional facts that are contained in the next results.

Lemma 2.2. Let $\mathcal{A}$ with derivations $D_{1}^{\mathcal{A}}, \ldots, D_{n}^{\mathcal{A}}$ be a model of $L D F_{0}$ and assume that the $D_{i}^{\mathcal{A}}$ are linearly independent over $\mathcal{A}$. Then there exist $a_{i, j} \in \mathcal{A}$ such that the derivations $\bar{D}_{i}^{\mathcal{A}}=\sum_{j} a_{i, j} D_{j}^{\mathcal{A}}$ are linearly independent over $\mathcal{A}$ and commute.

Proof. We shall assume $\mathcal{A}$ is a field and follow the proof of Proposition 6 in Chapter $0, \S 5$ of [1], with a few small modifications. As noted in the Introduction, this is an algebraic version of the Frobenius Theorem.

Let $\mathcal{C}=\bigcap_{i=1}^{n} \operatorname{Ker} D_{i}^{\mathcal{A}}$. Let $\left\{x_{i}\right\}_{i \in I}$ be a transcendence basis of $\mathcal{A}$ over $\mathcal{C}$. Let $\delta_{i}$ be the unique derivation on $\mathcal{A}$ satisfying $\delta_{i}(c)=0$ for all $c \in \mathcal{C}$ and $\delta_{i}\left(x_{j}\right)=1$ if $i=j$ and 0 if $i \neq j$. Any $x \in \mathcal{A}$ will lie in a subfield algebraic over $\mathcal{C}\left(x_{1}, \ldots, x_{N}\right)$ for some $N$, and so $\delta_{i}(x)=0$ for all $i>N$. For any derivation $D$ of $\mathcal{A}$ that is trivial on $\mathcal{C}$, consider the sum $\sum_{i \in I} D\left(x_{i}\right) \delta_{i}$. Although this sum is infinite, the previous remark shows that for any $x \in \mathcal{A}$, the sum $\sum_{i \in I} D\left(x_{i}\right) \delta_{i}$ makes sense and, using the results of Chapter VII, $\S 5$ of [2], one can show that $D=\sum_{i \in I} D\left(x_{i}\right) \delta_{i}$. 
Since the $D_{i}$ are linearly independent over $\mathcal{A}$ there exist $a_{i, j} \in \mathcal{A}$ such that the derivations $\bar{D}_{i}^{\mathcal{A}}=\sum_{j} a_{i, j} D_{j}^{\mathcal{A}}$ satisfy (after a possible renumbering of the $\delta_{j}$ )

$$
\bar{D}_{i}^{\mathcal{A}}=\delta_{i}\left(\bmod \sum_{j>n} \mathcal{A} \delta_{j}\right)
$$

for $i=1, \ldots, n$. To see that the $\bar{D}_{i}^{\mathcal{A}}$ are linearly independent over $\mathcal{A}$ note that if $\sum_{j} b_{j} \bar{D}_{j}^{\mathcal{A}}=0$, then $0=\sum_{j} b_{j} \bar{D}_{j}^{\mathcal{A}}\left(x_{i}\right)=b_{i}$. We now claim that the $\bar{D}_{i}^{\mathcal{A}}$ commute. Since the $\delta_{i}$ commute, we have that

$$
\left[\bar{D}_{r}^{\mathcal{A}}, \bar{D}_{s}^{\mathcal{A}}\right]=0\left(\bmod \sum_{i>n} \mathcal{A} \delta_{i}\right)
$$

for any $r, s$. Since the $\mathcal{A}$-span of the $\bar{D}_{i}^{\mathcal{A}}$ is closed under [, ], we have that there exist $b_{r, s, j} \in \mathcal{A}$ such that

$$
\begin{aligned}
{\left[\bar{D}_{r}^{\mathcal{A}}, \bar{D}_{s}^{\mathcal{A}}\right] } & =\sum_{j=1}^{n} b_{r, s, j} \bar{D}_{j}^{\mathcal{A}} \\
& =\sum_{j=1}^{n} b_{r, s, j} \delta_{j}\left(\bmod \sum_{i>n} \mathcal{A} \delta_{i}\right) .
\end{aligned}
$$

Therefore, we have that $b_{r, s, j}=0$ for all $r, s$, and $j$, and hence that the $\bar{D}_{j}^{\mathcal{A}}$ commute.

We shall need to compare Lie rings for two different Lie algebras $\mathcal{L}_{1}$ and $\mathcal{L}_{2}$. We will denote by $L D F_{0}^{1}$ (resp. $L D F_{0}^{2}$ ) the theory of Lie rings based on the action of the Lie algebra $\mathcal{L}_{1}$ (resp. $\mathcal{L}_{2}$ ). We will assume the two algebras are of the same dimension over $\mathcal{F}$.

Lemma 2.3. Let $\mathcal{A}$ with derivations $D_{1}^{1}, \ldots, D_{n}^{1}$ be a model of $L D F_{0}^{1}$ and assume that the $D_{i}^{1}$ are linearly independent over $\mathcal{A}$. For $i=1, \ldots, n$, let $D_{i}^{2}=\sum_{j} a_{i, j} D_{j}^{1}$ for some $a_{i, j} \in \mathcal{A}$ and assume that $\mathcal{A}$ with derivations $D_{1}^{2}, \ldots, D_{n}^{2}$ is a model of $L D F_{0}^{2}$. If $\mathcal{B}$ is an extension of $\mathcal{A}$ that is a model of $L D F_{0}^{1}$ with respect to the extensions of the derivations $D_{1}^{1}, \ldots, D_{n}^{1}$, then the formulas $D_{i}^{2}=\sum_{j} a_{i, j} D_{j}^{1}$ define derivations on $\mathcal{B}$ such that $\mathcal{B}$ with these derivations is a model of $L D F_{0}^{2}$.

Proof. Let $\alpha_{k, l}^{m}$ be the structure constants of $\mathcal{L}_{1}$ and let $\beta_{k, l}^{m}$ be the structure constants of $\mathcal{L}_{2}$. We have

$$
\begin{aligned}
{\left[D_{l}^{2}, D_{k}^{2}\right] } & =\sum_{j}\left(\sum_{i}\left(a_{l, i} D_{i}^{1}\left(a_{k, j}\right)-a_{k, i} D_{i}^{1}\left(a_{l, j}\right)\right)+\sum_{r, s} a_{l, r} a_{k, s} \alpha_{r, s}^{j}\right) D_{j}^{1}, \text { and } \\
\sum_{m} \beta_{l, k}^{m} D_{m}^{2} & =\sum_{j}\left(\sum_{m} \beta_{l, k}^{m} a_{m, j}\right) D_{j}^{1} .
\end{aligned}
$$

First note that since the $D_{j}^{1}$ are linearly independent over $\mathcal{A}$, these derivations are linearly independent over $\mathcal{B}$ as well. We therefore have that $\left[D_{l}^{2}, D_{k}^{2}\right]=\sum_{m} \beta_{l, k}^{m} D_{m}^{2}$ if and only if

$$
\sum_{i}\left(a_{l, i} D_{i}^{1}\left(a_{k, j}\right)-a_{k, i} D_{i}^{1}\left(a_{l, j}\right)\right)+\sum_{r, s} a_{l, r} a_{k, s} \alpha_{r, s}^{j}=\sum_{m} \beta_{l, k}^{m} a_{m, j}
$$

for all $j$. If this holds in $\mathcal{A}$, then it will hold in $\mathcal{B}$. 
In particular, if the basis elements $D_{i}^{2}$ commute, we have

Corollary 2.4. Let $\mathcal{A}$ with derivations $D_{1}^{1}, \ldots, D_{n}^{1}$ be a model of $L D F_{0}$ and assume that the $D_{i}^{1}$ are linearly independent over $\mathcal{A}$. For $i=1, \ldots, n$, let $D_{i}^{2}=\sum_{j} a_{i, j} D_{j}^{1}$ for some $a_{i, j} \in \mathcal{A}$ and assume that the $D_{i}^{2}$ commute as derivations on $\mathcal{A}$. If $\mathcal{B}$ is an extension of $\mathcal{A}$ that is a model of $L D F_{0}$, then the $D_{i}^{2}$ commute as derivations on $\mathcal{B}$.

Note that in model theoretic terms, the statement that the basis elements $D_{i}^{2}$ commute, which a priori is a universal statement, is actually (equivalent to) a quantifier-free statement, given that the $D_{i}^{1}$ are linearly independent.

\section{Axioms For the Model COMPletion of $L D F_{0}$}

Let us begin by recalling the situation for fields with commuting derivations. In 3], McGrail gave axioms for the model completion $m$-DCF of the universal theory $m$-DF of differential fields with $m$ commuting derivations $D_{1}, \ldots, D_{n}$. She also showed that this former theory has elimination of quantifiers. In particular, for any field $F$ with $m$ commuting derivations and any system

$$
\begin{aligned}
\mathcal{S}\left(u_{1}, \ldots, u_{r}, v_{1}, \ldots, v_{s}\right) & =\left\{f_{1}\left(u_{1}, \ldots, u_{r}, v_{1}, \ldots, v_{s}\right)\right. \\
& =0, \ldots, f_{t}\left(u_{1}, \ldots, u_{r}, v_{1}, \ldots, v_{s}\right) \\
& \left.=0, g\left(u_{1}, \ldots, u_{r}, v_{1}, \ldots, v_{s}\right) \neq 0\right\}
\end{aligned}
$$

of differential polynomials, there exist systems $\mathcal{T}_{1}\left(u_{1}, \ldots, u_{r}\right), \ldots, \mathcal{T}_{l}\left(u_{1}, \ldots, u_{r}\right)$ such that for any $u_{1}, \ldots, u_{r} \in F$ there exists a solution $v_{1}, \ldots, v_{s}$ in some differential extension of $F$ if and only if the $u_{i}$ satisfy one of the systems $\mathcal{T}_{i}\left(u_{1}, \ldots, u_{r}\right)$.

Let $\mathcal{F}, \mathcal{L}$ and $\phi_{\mathcal{F}}$ be as above. Let $\mathcal{R}_{\mathcal{F}}$ be the ring of normal polynomials with coefficients in $\mathcal{F}$. For any $t \in \mathbb{N}$, we will denote by $\mathcal{R}_{\mathcal{F}}\left[x_{1}, \ldots, x_{t}\right]$ the usual (not differential) ring of polynomials in the variables $x_{1}, \ldots, x_{t}$ with coefficients in $\mathcal{R}_{\mathcal{F}}$. The axioms for $L D C F_{0}$, the theory of Lie differentially closed fields of characteristic zero, are the axioms for $L D F_{0}$ plus the axioms for fields and the following axioms:

(1) for any $t$ and any polynomial $p\left(x_{1}, \ldots, x_{t},\left\{X_{I}\right\}\right) \in \mathcal{R}_{\mathcal{F}}\left[x_{1}, \ldots, x_{t}\right]$ we have an axiom that states that for any $a_{1}, \ldots, a_{n}$ such that $p\left(a_{1}, \ldots, a_{n},\left\{X_{I}\right\}\right) \neq$ 0 there exists $b$ such that $p\left(a_{1}, \ldots, a_{n},\left\{D^{I}(b)\right\}\right) \neq 0$;

(2) there exists an $n^{2}$-tuple of elements $\mathbf{x}=\left(x_{1,1}, \ldots, x_{n, n}\right)$ such that the derivations $D_{1}^{\mathbf{x}}=\sum_{j} x_{1, j} D_{j}, \ldots, D_{n}^{\mathbf{x}}=\sum_{j} x_{n, j} D_{j}$ form a linearly independent set of commuting derivations;

(3) for any $\mathbf{x}$ such that $\left\{D_{1}^{\mathbf{x}}, \ldots, D_{n}^{\mathbf{x}}\right\}$ is a linearly independent set of commuting derivations and any system $S\left(u_{1}, \ldots, u_{n}, v_{1}, \ldots, v_{m}\right)$ involving differential polynomials in the $D_{i}^{\mathbf{x}}$, we have

$$
\exists u_{1}, \ldots, v_{m} \mathcal{S}\left(u_{1}, \ldots, u_{n}, v_{1}, \ldots, v_{m}\right)
$$

if and only if

$$
\bigvee_{j=1}^{l} \mathcal{T}_{j}\left(u_{1}, \ldots, u_{n}\right)
$$

where the $\mathcal{T}_{j}$ are as described above.

Note that (3) implies that the models of this theory are algebraically closed.

We will use Blum's criteria ( $[5$, Section 17$)$ to show that these axioms give the model completion of $L D F_{0}$. The first step is to show that any model $\mathcal{A}$ of $L D F$ can 
be extended to a model of $L D C F_{0}$. Taking quotient fields of the ring constructed in Lemma 2.1 we extend $\mathcal{A}$ to a model $K^{0}$ of $L D F_{0}$ were the derivations are linearly independent over $K^{0}$. Note that the ring constructed in Lemma 2.1 allows us to conclude that $K^{0}$ satisfies axiom scheme (1). Lemma 2.2 implies that axiom (2) holds. Let $\mathcal{N}_{0}$ be the set of $n^{2}$-tuples of elements in $K^{0}$ and assume that this set is well ordered. Let $\mathbf{x}$ be the smallest element of $\mathcal{N}_{0}$ such that $\left\{D_{1}^{\mathbf{x}}, \ldots, D_{n}^{\mathbf{x}}\right\}$ is a linearly independent set of commuting derivations and let $K_{1}^{0}$ be the differential closure of $K^{0}$ thought of as a field with commuting derivations $\left\{D_{1}^{\mathbf{x}}, \ldots, D_{n}^{\mathbf{x}}\right\}$. Since the $D_{i}$ can be expressed a $K^{0}$-linear combinations of the $D_{i}^{\mathbf{x}}$, Lemma 2.3 implies that $K_{1}^{0}$ is still a model of $L D F_{0}$. Let $\overline{\mathbf{x}}$ be the next smallest element of $\mathcal{N}_{0}$ such that $\left\{D_{1}^{\overline{\mathrm{x}}}, \ldots, D_{n}^{\overline{\mathrm{x}}}\right\}$ is a linearly independent set of commuting derivations and let $K_{2}^{0}$ be the differential closure of $K_{1}^{0}$ thought of as a field with commuting derivations $\left\{D_{1}^{\overline{\mathrm{x}}}, \ldots, D_{n}^{\overline{\mathrm{x}}}\right\}$. We can continue in this way for all elements of $\mathcal{N}_{0}$ and, taking unions, form a field $\bar{K}^{0}$ such that for any $\mathbf{x}$ in $\mathcal{N}_{0}$ such that $\left\{D_{1}^{\mathbf{x}}, \ldots, D_{n}^{\mathbf{x}}\right\}$ is a linearly independent set of commuting derivations, $\bar{K}^{0}$ contains the differential closure of $K^{0}$ thought of as a field with commuting derivations $\left\{D_{1}^{\mathbf{x}}, \ldots, D_{n}^{\mathbf{x}}\right\}$. Again Lemma 2.3 implies that $\bar{K}^{0}$ is a model of $L D F_{0}$. We let $K^{1}$ be the quotient field of $\mathcal{R}_{\bar{K}^{0}}$. Note that $K^{1}$ satisfies axiom scheme (1). We now repeat this process and form a field $K^{2}$ such that for any $n^{2}$-tuple $\mathbf{x}$ of elements in $K^{1}$ such that $\left\{D_{1}^{\mathbf{x}}, \ldots, D_{n}^{\mathbf{x}}\right\}$ is a linearly independent set of commuting derivations, $K^{2}$ contains the differential closure of $K^{1}$ thought of as a field with commuting derivations $\left\{D_{1}^{\mathbf{x}}, \ldots, D_{n}^{\mathbf{x}}\right\}$ and also satisfies axiom scheme (1). One now sees that $K^{\infty}=\bigcup K^{i}$ is a model of $L D F_{0}$ and satisfies the axioms of $L C D F_{0}$.

We will now show that if $\mathcal{A}=L D F_{0}, \mathcal{B}=L D C F_{0}, \mathcal{B}$ being $|\mathcal{A}|^{+}$-saturated and $\mathcal{A} \subset \mathcal{B}$, then for any simple extension $\mathcal{A}(a)$ there is an $\mathcal{A}$-embedding $f: \mathcal{A}(a) \rightarrow \mathcal{B}$. We will first show that we can assume that $\mathcal{A}$ satisfies axioms (1) and (2) above.

Let $\mathcal{R}_{\mathcal{A}(a)}=\mathcal{A}(a)\left(\left\{X_{I}\right\}\right)$ be the field fractions of normal polynomials over $\mathcal{A}(a)$. Axiom (1) and saturation imply that there is an $\mathcal{A}$-embedding of $\mathcal{A}\left(\left\{X_{I}\right\}\right)$ into $\mathcal{B}$. Furthermore, $\mathcal{A}\left(\left\{X_{I}\right\}\right)$ satisfies axiom schemes (1) and (2). If we can extend this embedding to $\mathcal{A}\left(\left\{X_{I}\right\}\right)(a)$, then restricting to $\mathcal{A}(a)$ will give the desired conclusion. We will therefore assume from the beginning that $\mathcal{A}$ satisfies axiom schemes (1) and (2).

Let $\mathrm{x}$ be elements of $\mathcal{A}$ such that $D_{i}^{\mathrm{x}}$ are linearly independent commuting derivations. Corollary 2.4 implies that these derivations commute on $\mathcal{A}(a)$ as well. The isomorphism type of $a$ over the field $\mathcal{A}$ with derivations $\left\{D_{i}^{\mathbf{x}}\right\}$ is determined by the set of $\left\{D_{i}^{\mathbf{x}}\right\}$-differential polynomials that $a$ satisfies and by the set that it does not satisfy. By axiom scheme (3) and saturation, we can realize this type in $\mathcal{B}$ and therefore get an embedding of $\mathcal{A}(a)$ into $\mathcal{B}$, considered as $\left\{D_{i}^{\mathbf{x}}\right\}$-differential fields. Since the $\left\{D_{i}\right\}$ are linear combinations of the $\left\{D_{i}^{\mathbf{x}}\right\}$, this is also an embedding as models of $L D F_{0}$.

\section{ACKNOWLEDGMENTS}

The author would like to thank Evelyne Hubert for several useful discussions and especially for pointing out the relation between the result of Cassidy and Kolchin appearing as Proposition 6, p. 12 of [1] and the Frobenius Theorem. He would also like to thank Yoav Yaffe for discussions that helped clarify his initially vague ideas and the anonymous referee for useful comments. 


\section{REFERENCES}

[1] Ellis R. Kolchin, Differential algebraic groups, Pure and Applied Mathematics, vol. 114, Academic Press Inc., Orlando, FL, 1985. MR0776230 (87i:12016)

[2] Serge Lang, Algebra, 3rd ed., Addison-Wesley, New York, 1993. MR1878556 (2003e:00003)

[3] Tracey McGrail, The model theory of differential fields with finitely many commuting derivations, J. Symbolic Logic 65 (2000), no. 2, 885-913. MR.1771092 (2001h:03066)

[4] David Pierce, Differential forms in the model theory of differential fields, J. Symbolic Logic 68 (2003), no. 3, 923-945. MR2000487 (2004h:03080)

[5] Gerald E. Sacks, Saturated model theory, W. A. Benjamin, Inc., Reading, Mass., 1972, Mathematics Lecture Note Series. MR0398817(53:2668)

[6] Veeravalli S. Varadarajan, Lie Groups, Lie Algebras, and their Representations, Graduate Texts in Mathematics, no. 102, Springer-Verlag, New York, 1984. MR0746308 (85e:22001)

[7] Yoav Yaffe, Model completion of Lie differential fields, Ann. Pure Appl. Logic 107 (2001), no. 1-3, 49-86. MR1807840 (2003i:03040)

Department of Mathematics, North Carolina State University, Box 8205, Raleigh, North Carolina 27695-8205

E-mail address: singer@math.ncsu.edu 